\title{
Nova combinação e chave revisada para Dendrophorbium (Asteraceae - Senecioneae) no Brasil
}

A new combination and revised key to the Brazilian species of

Dendrophorbium (Asteraceae-Senecioneae)

Aristônio M. Teles ${ }^{1}$

\begin{abstract}
Resumo
Uma nova combinação é proposta para a tribo Senecioneae (Asteraceae) no Brasil: Dendrophorbium subnemoralis (Dusén) A.Teles, além disso, é apresentada uma chave revisada para identificação de espécies do gênero ocorrentes no Brasil.

Palavras-chave: combinação nova, Compositae, Senecio.
\end{abstract}

\section{Abstract}

A new combination is proposed for the tribe Senecioneae (Asteraceae) in Brazil: Dendrophorbium subnemoralis (Dusén) A.Teles. Furthermore, a revised key to the Brazilian species of Dendrophorbium is presented.

Key words: new combination, Compositae, Senecio.

\section{Introdução}

Dendrophorbium (Cuatrec.) C.Jeffrey é um gênero predominantemente andino (Jeffrey 1992), com aproximadamente 75 espécies que ocorrem na Argentina, Bolívia, Brasil, Paraguai e Venezuela (Nordenstam 2007). O gênero tal como é conhecido hoje foi descrito inicialmente como uma seção de Senecio (Senecio sect. Dendrophorbium Cuatrec.) (Nordenstam 1996). No entanto, Jeffrey (1992) elevou a seção à categoria de gênero para agrupar as espécies caracterizadas por apresentarem folhas grandes e geralmente dentadas, capitulescência corimbiforme a paniculiforme, capítulos radiados, 8 13 brácteas involucrais glabras, ramos do estilete truncado, obtuso ou cônico e cipselas 5-8 costadas, com carpopódio anuliforme (Nordenstam 1996; 2007).

As espécies de Dendrophorbium ocorrentes no Brasil foram agrupadas por Cabrera (1957) em Senecio sect. Myriocephalus Cabrera, porém quando Jeffrey (1992) elevou Dendrophorbium à categoria taxonômica de gênero, sinonimizou a seção Myriocephalus em Dendrophorbium e propôs novas combinações no referido gênero. Hind (1993) citou para o Brasil nove espécies do gênero. No entanto, Matzenbacher \& Baptista (1997) propuseram uma nova combinação em Dendrophorbium, e Teles et al. (2006) descreveram uma nova espécie para o gênero, elevando o número de espécies conhecidas no Brasil para 11. Pelser et al. (2007) demonstraram através de marcadores moleculares ( $n r I T S)$, que algumas espécies atualmente tratadas como Senecio, entre elas Senecio subnemoralis Dusén, deveriam ser incluídas em Dendrophorbium.

Baseado nas características morfológicas e nas informações prévias de estudos filogenéticos (Pelser et al. 2007) propõe-se uma nova combinação de Senecio subnemoralis Dusén em Dendrophorbium. De forma complementar apresenta-se uma chave para as espécies do gênero ocorrentes no Brasil.

\section{Resultados e Discussão}

Dendrophorbium subnemoralis (Dusén) A.Teles, comb. nov. Senecio subnemoralis Dusén, Arq. Mus. Nac. Rio Janeiro 13: 22. 1905.Tipo: BRASIL. SANTA CATARINA: perto da costa da Serra Geral, IV.1891, fl. e fr., E. Ule 1773 (holótipo R, foto!).

Senecio melloi Cabrera, Not. Mus. La Plata 15: 92. 1950, nom. superfl. Tipo: BRASIL. SANTA CATARINA: perto da costa da Serra Geral, IV.1891, fl. e fr., E. Ule 1773 (holótipo R).

Universidade Federal de Goiás, Instituto de Ciências Biológicas, Depto. Biologia Geral, Campus Samambaia, C.P. 131, 74001-970, Goiânia, GO, Brasil. 
Material adicional examinado: PARANÁ: São José dos Pinhais, Rio Pequeno, 4.III.1980, fl. e fr., $G$. Hatschbach 42768 (MBM, UEC). RIO GRANDE DO SUL: Veranópolis, em beira de estrada, IX.1993, fl. e fr., M. Sobral \& C. Miró 7668 (MBM). SANTA CATARINA: Braço do Norte, Urubici, Serra do Corvo Branco, 27.II.1996, fl. e fr., J.A. Jarenkow \& M. Sobral 3078 (MBM); Urubici, Serra do Corvo Branco, 17.II.1995, fl. e fr., G. Hatschbach \& O.S. Ribas 61717 (MBM); São Joaquim, Rodovia Urubici, São Joaquim, Rio Pericó, 18.VIII.2006, fl. e fr., G. Hatschbach \& E. Barbosa 79598 (MBM).

Senecio subnemoralis foi agrupado por Cabrera (1957) em Senecio sect. Myriocephalus.
No entanto, quando Jeffrey (1992) elevou as espécies desta seção à categoria de gênero, não propôs a combinação desta espécie em Dendrophorbium, provavelmente por não ter visto o holótipo que se encontra depositado no Herbário do Museu Nacional do Rio de Janeiro (R). A espécie, ora em questão, enquadra-se perfeitamente na circunscrição morfológica de Dendrophorbium. Além disso, resultados de estudos filogenéticos baseados em dados moleculares já demonstraram que esta espécie inclui-se no clado formado pelas espécies de Dendrophorbium (Pelser et al. 2007).

\section{Chave de identificação para as espécies de Dendrophorbium ocorrentes no Brasil (revisada a partir de Cabrera (1957))}

1. Folhas densamente tomentosas na face abaxial.

2. Árvores; flores do raio com limbo reduzido D. glaziovii (Baker) C.Jeffrey

2'. Ervas robustas ou arbustos; flores do raio com limbo desenvolvido.

3. Arbustos; folhas lanceoladas D. brachycodon (Baker) C.Jeffrey

3'. Ervas robustas; folhas ovadas a cordiformes.

4. Margem foliar crenulada; pecíolos não auriculados; flores do disco 30-35; corola 7-9 mm compr. D. restingae A.Teles, J.N.Nakaj. \& Stehmann

4'. Margem foliar denteada; pecíolos auriculados; flores do disco 22-25; corola 3,5-5 mm compr. D. paranense (Malme) Matzenb. \& Baptista

1'. Folhas glabras ou apenas laxamente pubescentes, ou lanuginosas na face abaxial.

5. Lâminas foliares quatro vezes mais compridas que largas.

6. Folhas oblanceoladas; pecíolos ca. $5 \mathrm{~mm}$ compr.; capitulescência fastigiata

D. fastigiaticephalum (Cabrera) C.Jeffrey

6'. Folhas lanceoladas ou oblongas; pecíolos 10-25 mm compr.; capitulescência corimbiforme ou paniculiforme.

7. Capitulescência corimbiforme; brácteas involucrais 8, 5-5,5 mm compr. .....

D. subnemoralis (Dusén) A.Teles

7'. Capitulescência paniculiforme; brácteas involucrais 12, 10-11 mm compr.

\section{D. bradei (Cabrera) C.Jeffrey}

5'. Lâminas foliares menos que quatro vezes mais compridas que largas.

8. Folhas estreitamente elípticas ou largamente ovadas, laxamente pubescentes ou laxamente lanuginosas na face abaxial.

9. Folhas estreitamente elípticas, $7-12 \times 5-7 \mathrm{~cm}$, laxamente pubescentes na face abaxial .... D. pluricephalum (Cabrera) C.Jeffrey

9'. Folhas largamente ovadas, ca. $35 \times 20 \mathrm{~cm}$, laxamente lanuginosas na face abaxial ..... D. catharinense (Dusén ex Cabrera) C.Jeffrey

8'. Folhas lanceoladas ou ovado-lanceoladas, glabras na face adaxial.

10. Folhas lanceoladas, 1,2-2,5 cm larg., 8-10 dentes em cada lado da margem D. limosum C.Jeffrey

10'. Folhas ovado-lanceoladas, 3-4,5 cm larg., 18-35 dentes em cada lado da margem.

11. Folhas com base arredondada ....... D. pellucidinerve (Sch.Bip. exBaker) C.Jeffrey 11'. Folhas com base cuneada D. fruticosum (Vell.) C.Jeffrey 


\section{Agradecimentos}

Agradeço a Mariana Machado Saavedra (JBRJ) o envio da fotografia do tipo de Senecio subnemoralis.

\section{Referências}

Cabrera, A.L. 1957. El genero Senecio (Compositae) en Brasil, Paraguay y Uruguay. Arquivos do Jardim Botânico do Rio de Janeiro 15: 163-264.

Jeffrey, C. 1992. The tribe Senecioneae (Compositae) in the Mascarene Islands with an annotated world check-list of the genera of the tribe. Notes on Compositae: VI. Kew Bulletin 47: 49-109.

Hind, D.J.N. 1993. A checklist of the Brazilian Senecioneae (Compositae). Kew Bulletin 48: 279-295.

Matzenbacher, N.I. \& Baptista, L.R.M. 1997. Uma nova combinação no gênero Dendrophorbium (Cuatrec.) C. Jeffrey (Asteraceae - Senecioneae).
Boletim do Museu Botânico Prefeitura Municipal de Curitiba 65: 1-3.

Nordenstam, B. 1996. Recent revision of Senecioneae and Calenduleae systematics. In: Hind, D.J.N. \& Beentje, H.J. (eds.). Compositae: Systematics. Vol. 1.Proceedings of the International Compositae Conference. Royal Botanic Gardens, Kew. Pp. 591-596.

Nordenstam, B. 2007. XII. The tribe Senecioneae Cass. 1819. In: Kadereit, J.W. \& Jeffrey, C. (eds.). The families and genera of vascular plants. Flowering plants, Eudicots, Asterales. Vol. 8. Springer, Berlin. Pp. 208-241.

Pelser, P.B.; Nordenstam, B.; Kadereit, J.W. \& Watson, L.E. 2007. An ITS phylogeny of tribe Senecioneae (Asteraceae) and a new delimitation of Senecio L. Taxon 56: 1077-1104.

Teles, A.M.; Nakajima, J.N. \& Stehmann, J.R. 2006. Dendrophorbium restingae (Asteraceae: Senecioneae), a new species from São Paulo, Brazil. Sida 22: 123-128. 